\title{
Finite density QCD with heavy quarks
}

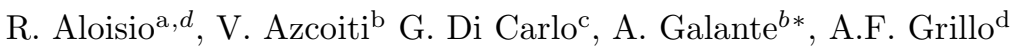 \\ aDipartimento di Fisica, Universitá dell'Aquila, via Vetoio, 67100 L'Aquila, Italy \\ bepartamento de Física Teórica, Facultad de Ciencias,Universidad de Zaragoza,50009 Zaragoza,Spain \\ ${ }^{\mathrm{c} I s t i t u t o}$ Nazionale di Fisica Nucleare, Laboratori Nazionali di Frascati, P.O.B. 13, 00044 Frascati, Italy \\ ${ }^{\text {d} I s t i t u t o ~ N a z i o n a l e ~ d i ~ F i s i c a ~ N u c l e a r e, ~ L a b o r a t o r i ~ N a z i o n a l i ~ d e l ~ G r a n ~ S a s s o, ~} 67010$ Assergi, Italy
}

In the large fermion mass limit of QCD at finite density the structure of the partition function greatly simplifies and can be studied analytically. We show that, contrary to general wisdom, the phase of the Dirac determinant is relevant only at finite temperature and can be neglected for zero temperature fields.

\section{Introduction}

Finite density QCD is still a poorly understood field from an analytical point of view. We miss any quantitative data about the passage from the hadronic to the quark-gluon plasma phase at high densities and even more qualitative information like the order of the phase transition (if any) is not available.

This situation mainly reflects the difficulties to perform numerical simulations of QCD at finite chemical potential $\mu$. Almost all the standard algorithms for zero density QCD include the fermionic determinant in the generation of configurations and fail for complex valued determinants. Attempts to create ad hoc algorithms have been made but they are generally very computer consuming, they rely on some a priori unjustified approximations to get real valued observables and the results obtained up to now are plagued from unphysical effects like the early onset of the baryon density [1], [2].

The main difficulty is to evaluate correctly the contribution of the phase of the action. In fact the partition function can be written as the product of two terms:

$$
\mathcal{Z}=\mathcal{Z}_{\|}\langle\cos \phi\rangle_{\|}
$$

where the first is evaluated using the modulus of the Dirac determinant and the second is the mean

\footnotetext{
*Talk presented by A. Galante
}

value of the cosine of the phase calculated weighting the configurations with the usual pure gauge part of the action times the modulus of the determinant. In the thermodynamic limit $\langle\cos \phi\rangle_{\|}$ gives a finite contribution to the free energy density if it is proportional to $e^{-V}$. If this is the case there is no chance to determine numerically its value: for any reasonable statistic, is not possible to evaluate it from a set of measurements of $O(1)$.

The simple idea to consider only the modulus of the action has received very little attention, especially after the studies on the random matrix model where the phase of the Dirac determinant is fundamental for obtaining the correct result [3]. In QCD the one dimensional case can be addressed analytically and we can see that the average value of the cosine of the phase goes to 1 as we approach the thermodynamic limit [4]; the physically relevant four dimensional theory can only be studied in the large bare mass limit where several simplifications in the fermionic determinant structure occur.

\section{The strong coupling limit}

We can first write the fermionic matrix $\Delta$ separating the contribution of forward (backward) temporal links $G\left(G^{\dagger}\right)$ and spatial links $V$ :

$2 \Delta=2 m I+e^{\mu} G+e^{-\mu} G^{\dagger}+V$ 
When $m \gg 1$ only the mass term and the forward temporal links can contribute to the fermionic determinant. Since quarks do not propagate $\operatorname{det} \Delta$ can be written as a function of traces of Polyakov loops $\left(L_{i}\right)$ only and we get, except for an irrelevant multiplicative constant,

$\operatorname{det} \Delta=e^{3 V_{s} L_{t} \mu} \prod_{i=1}^{V_{s}}\left(c^{3}+c^{2} \operatorname{Tr} L_{i}+c \operatorname{Tr} L_{i}^{*}+1\right)(3)$

where $V_{s}$ is the spatial volume, $L_{t}$ the lattice temporal extent and $c=\left(\frac{2 m}{e^{\mu}}\right)^{L_{t}}[5]$.

In the strong coupling limit we can solve exactly the theory since the Polyakov lines are independent and the partition function factorizes trivially:

$\mathcal{Z}(\beta=0)=e^{3 V_{s} L_{t} \mu}\left(c^{3}+1\right)^{V_{s}}$

It follows immediately that, in the thermodynamic limit, the system undergoes a first order transition at $\mu_{c}=\log 2 m$ where the baryon density jumps from zero to the saturation value. Whatever the spatial volume is the singular behaviour in the free energy density is related to $L_{t}$ only.

Regarding equation (17) we see that an upper bound for $\mathcal{Z}_{\|}$is given by the partition function at $\beta=\infty$ and therefore

$\langle\cos \phi\rangle_{\|} \geq\left(\frac{c^{3}+1}{(c+1)^{3}}\right)^{V_{s}}$

As the volume goes to infinity this ratio goes to 1 except when $\mu=\mu_{c}$ where it goes to zero exponentially with the spatial volume; we have a correct description of our zero temperature system replacing the fermion determinant by its absolute value.

The situation changes completely if we consider the effect of non zero temperature: we must keep $L_{t}$ finite and consider the limit of infinite spatial volume. From (14) we see that the transition becomes a smooth crossover and, from the definition (1), we can also realize easily that $\langle\cos \phi\rangle_{\|} \sim e^{-V_{s}}$. Since the system volume is proportional to $V_{s}$ this implies that the phase can not be neglected.

The availability of the analytical solution (4) can also provide a useful tool to check the results of numerical simulations. Similarly to the

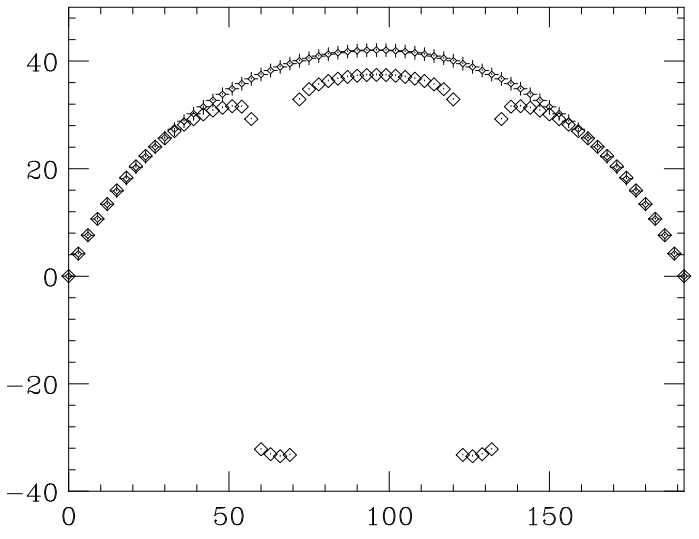

Figure 1. Logarithm of the modulus of the GCPF coefficients times the sign of the coefficients as a function of the coefficient order: analytic (crosses) and numerical (diamonds) results for $V=4^{3} \times 4$.

Grand Canonical Partition Function formalism we have written the partition function $\mathcal{Z}$ as a polynomial in the variable $c$ and studied the convergence properties of the coefficients. We generated several thousands of random gauge configurations and calculated the averaged expansion coefficients. This has been done for lattices $4^{4}$ (fig. 1), $4^{3} \times 20$ (fig. 2) and $10^{3} \times 4$ (fig. 3) and results are plotted, as a function of the coefficient order, superimposed to the ones obtained from (4). From the plots we can realize that $i$ ) numerical results are not positive definite as they should be but for large $L_{t}$ this phenomena disappears; $i i$ ) even considering the modulus of the averaged coefficients we do not get the correct order of magnitude in the central region; iii) increasing $L_{t}$ or $V_{s}$ the situation makes worse.

This seems to indicate that, in this model, our configuration ensemble is not relevant for the physics at intermediate $c($ i.e. $c \sim 1)$ and a correct determination of all the coefficients from a random ensemble is not possible. Increasing the temporal lattice extent the contribution of the phase becomes smaller and, with reasonable statistic, we get positive averaged coefficients. Neverthe- 


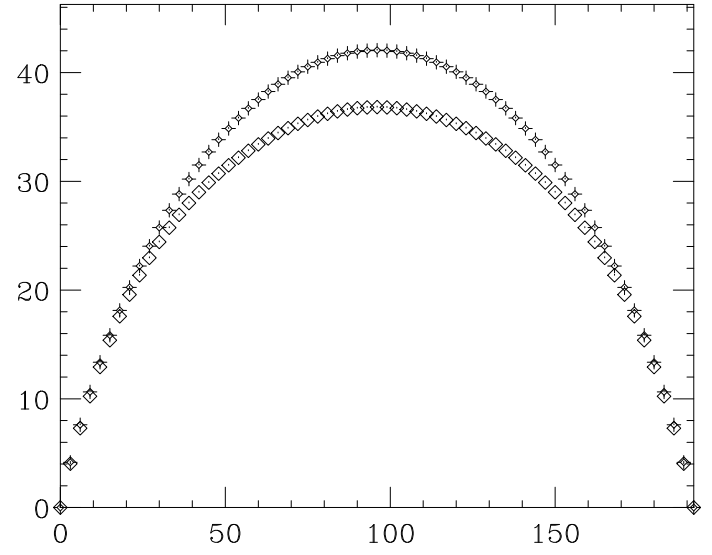

Figure 2. As in fig. 1 for $V=4^{3} \times 20$.

less their convergence to the correct values do not improve suggesting that a non accurate determination of $\langle\cos \phi\rangle_{\|}$may not be the only source of artifacts in simulations (see also [i]).

\section{Finite coupling}

At non zero $\beta$ the pure gauge part of the action couples the Polyakov lines among themselves and any trivial factorization in the partition function is not possible any more. The partition function can be written as the product of the pure gauge partition function times the $\beta=0$ partition function times a factor $R$ :

$R=\frac{\int[d U] e^{-\beta S_{G}(U)} \prod_{i=1}^{V_{s}}\left(1+\frac{c^{2} \operatorname{Tr} L_{i}+c \operatorname{Tr} L_{i}^{*}}{c^{3}+1}\right)}{\int[d U] e^{-\beta S_{G}(U)}}(6)$

From the previous section we conclude that, in the zero temperature case, the only possible contribution of the phase comes from (6). For large temporal extent $c$ approaches 0 or $\infty$ and in both cases the absolute value of all the factors in the numerator of (6) is upper and lower bounded by a finite number. This implies that $R$ behaves at most exponentially with the spatial lattice volume $V_{s}$ except for $\mu=\log 2 m$. When this happens the phase is clearly not relevant and the free energy density reduces to the pure gauge contribution plus the infinite coupling term: we

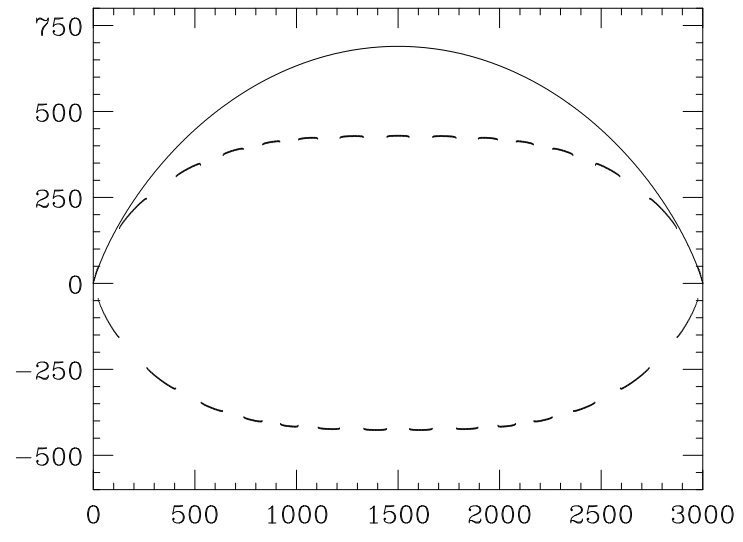

Figure 3. As in fig. 1: analytic (uppermost continuous line) and numerical results for $V=$ $10^{3} \times 4$.

get again a first order saturation transition at $\mu_{c}=\log 2 m$ for all the values of $\beta$.

At finite temperature the scenario is completely different. The non analyticity in the $\mathcal{Z}(\beta=0)$ disappears and expression (6) can also give a finite contribution in the free energy density. Even if an exact evaluation of $R$ is not possible this points toward a smooth crossover from the zero density to the saturation regime as soon as $L_{t}$ is considered finite in the thermodynamic limit.

\section{REFERENCES}

1. I.M. Barbour, S.E. Morrison, E.G. Klepfish, J.B. Kogut, M.P. Lombardo, Phys. Rev. D56, 7063 (1997); Nucl. Phys. Proc. Suppl. 60A (1998) 220.

2. R. Aloisio, V. Azcoiti, G. Di Carlo, A. Galante, A.F. Grillo, Phys. Lett. B428 (1998) 166; hep-lat/9804020 to appear in Phys. Lett. B.

3. M.A. Stephanov, Phys. Rev. Lett. 76 (1996) 4472.

4. R. Aloisio, V. Azcoiti, G. Di Carlo, A. Galante, A.F. Grillo, Nucl. Phys. Proc. Suppl. 63 (1998) 442.

5. T.C. Blum, J.E. Hetrick, D. Toussaint, Phys. Rev. Lett. 76 (1996) 1019. 